\title{
Shape Image Retrieval Using Elastic Matching Combined with Snake Model
}

\author{
Chen Sheng and Yang Xin \\ Institute of Image processing and pattern Recognition, \\ Shanghai Jiaotong University, Shanghai 200030, P. R. China \\ chnshnahotmail.com, yangxinasjtu.edu.cn
}

\begin{abstract}
Shape-based recovery from image or video databases has become an important information retrieval problem. It is particularly challenging, owning to the difficulty to derive a similarity measurement that closely conforms to the common perception of humans. The goal of the current work is to achieve idea retrieval accuracy with reasonable speed and support for partial and occluded shapes. So, in this paper we introduce the elastic matching that is inspired by Duncan and Ayache combined with snake as a new shape retrieval technique. The elastic matching is to minimize of a quadratic fitting criterion, which consists of a curvature dependent bending energy term and a smoothness term. To reduce the computational complexity, the equation corresponding is only to the minimization of one-dimensional fitting criterion. As a result, the method proposed has the advantage of retrieve resemble objects with reasonable speed and less training samples.
\end{abstract}

\section{Introduction}

The problem of similarity-based retrieval of visual shapes has been the subject of much research. Shape retrieval has practical interest for at least two reasons. First, there are many applications in which shapes can be extracted from images with high reliability. Second, there are many sources of visual shapes in addition to images; for example, computer graphics models, CAD models, geographical information systems, MPEG-7 objects, and drawing programs contain shapes or allow shapes to be synthesized from the stored data.

Two essential requirements must be met by a shape retrieval system: accuracy and efficiency. Different approaches make different tradeoffs among accuracy, efficiency, and other desirable characteristics, such as the ability to handle partial or occluded shapes. In this paper, the method is to emphasize accuracy while providing a reasonable level of speed, and also support for partial and occluded shapes.

There are many shape retrieval techniques in the literature: Flickner et al. [1,2] represent shapes by vectors of global feature. A $R^{*}$-tree multidimensional access method is used to find database feature vectors that are similar to the query feature vector. This approach is fast, but does not support partial or occluded shapes due to the dependence on global shape properties. Mokhtarian et al. [3] represent each significant segment of the contour by a point in the curvature scale-space representation of the 
contour. Matching is performed by aligning these point sets in curvature scale-space. Gdalyahu and Weinshall [4] match shape contours structurally. Each contour is represented as an attributed string corresponding to a sequence of contour segments; the attributed properties are geometric properties of segments. Syntactic matching is performed by computing the minimum edit distance between the strings. Structural shape matching methods are highly effective since they perform a global optimization that takes into account both structural and geometric information. In addition, partial and occluded shapes can be matched. The principal disadvantage of these approaches is that they are computationally expensive. Del Bimbo and Pala [5] integrate indexing with syntactic matching. In their approach, multi-scale representations of the database contours are stored as a graph. Given a query, sequences of segments are matched at the coarsest scale, and if the match is successful, then finer-scale matches are attempted. The method is not orientation invariant since the initial matching is based on segment orientation.

In this paper, a new shape retrieval method is introduced which propose a different elastic matching method. Combined with the snake model, the template is deformed to adjust itself to the shape images. The elastic deformation spent by the template to match the shape images and the matching degree are used to evaluate the similarity between them. It is scaling, rotation and translation invariant and can recover the missing part or remove the occluded part in the shapes. More significant, to reduce the computational complexity, the equation corresponding to the minimization of the fitting criterion has been interpreted as a simple form.

\section{The Approach of Shape Representation and Matching}

In this approach, suppose we have a one-dimensional shape template:

$$
C_{\text {query }}(s)=(x(s), y(s))(s \in[0,1])
$$

where: $s$ is the parameter of length along the template. Let $\Omega$ be a bounded open subset of $R^{2}$, with $\partial \Omega$ its boundary, we have a shape image $u_{0}: \bar{\Omega} \rightarrow R$. So our purpose is to search for a contour with a shape similar to the original template $C_{\text {query }}(s)$.

The template must warp taking into account two opposite requirements. First, it must match the edge of shape in images as closely as possible and the snake model [6] is introduced. It is to minimize the following energy functional:

$$
E_{\text {snake }}\left(u_{0}, C_{\text {deformed }}\left(s^{\prime}\right)\right)=E_{\text {int }}\left(C_{\text {deformed }}\left(s^{\prime}\right)\right)+E_{\text {ext }}\left(u_{0}, C_{\text {deformed }}\left(s^{\prime}\right)\right)
$$

where: $C_{\text {deformed }}$ is the deformed template shape with $S^{\prime}$ the parameter of length, $E_{\text {int }}$ is the internal energy that controls the smoothness of the shape and $E_{\text {ext }}$ is the external energy that attracts of the template evolving to the edge of object.

Secondly, it needs another energy to measure the deformation of the template also called elastic deformation by us. In this paper, we use a criterion to measure the de- 
formation, which consists of a curvature dependent bending energy term and a smoothness term.

The curvature is a key descriptor of the shape in this method because it satisfies the following requirements:

1. The curvature is invariant under rotation and translation.

2. The curvature is a local, scale-dependent feature. A series of shapes can be matched at any desired scale by using a multi-resolution approach.

These allow one to introduce a local bending energy measure of the form:

$$
E_{\text {curvature }}=\int\left(k_{C_{\text {deformed }}}\left(s^{\prime}\right)-k_{C_{\text {query }}}(s)\right)^{2} d s
$$

where: $k_{C_{\text {deformed }}}\left(s^{\prime}\right)$ is the curvature of deformed template $C_{\text {deformed }}$ at $s^{\prime}$ as well as $\boldsymbol{k}_{\boldsymbol{C}_{\text {query }}}(\boldsymbol{s})$.

We also wish the displacement vector field to vary smoothly along the contour [7]:

$$
E_{\text {smooth }}=\int\left\|\frac{\partial\left(C_{\text {deformed }}\left(s^{\prime}\right)-C_{\text {query }}(s)\right)}{\partial s}\right\| d s
$$

where: $\|\cdot\|$ denotes the norm associated to the scalar product $\langle\cdot, \cdot\rangle$ in the space $\mathfrak{R}^{2}$.

So the criterion is composed of two terms:

$$
E_{\text {elastic }}=E_{\text {curvature }}+\lambda E_{\text {smooth }}
$$

where $\lambda$ is a relative weighting factor (a high $\lambda$ value means heavy smoothing). Actually we have experimented different strategies for weighting the relative balance between smoothness and curvature similarity. One of the successful choices for $\lambda$ seems to be the heuristically defined adaptive weighting parameter as following [8]:

$$
\lambda=\frac{1}{1+k_{C_{\text {query }}}(s)}
$$

The idea underlying this parameter definition is to make $E_{\text {curvature }}$ preponderant for those contours having characteristic points of high curvature. In the opposite case, the above definition for the parameter $\lambda$ will make $E_{\text {smooth }}$ preponderant.

Duncan [7] finds a displacement field by direct minimization of a discrete form of Equation (5), the resulting displacement vectors in his approach may, however, map points not belonging to the two contours. This problem was solved by Cohen [9]. His mathematical model can be summarized as follow: Given two contours $C_{d e f o r m e d}$ and $C_{\text {query }}$ parameterized by $s^{\prime} \in[0,1]$ and $s \in[0,1]$, we have to determine a function $f:[0,1] \rightarrow[0,1] ; s^{\prime} \rightarrow s$ satisfying

$$
f(0)=0 \text { and } f(1)=1
$$


And

$$
f=\arg \min E_{\text {elastic }}(f)
$$

Cohen obtains the function $f$, which satisfies Equation (8) and conditions (7), by a variational method. This method finds a local minimum of the function $E_{\text {elastic }}(f)$ as the solution of the Eular-Lagrange equation $\nabla E_{\text {elastic }}(f)=0$ :

$$
\begin{aligned}
f^{\prime \prime}\left\|C_{\text {deformed }}(f)\right\|^{2}+k_{C_{\text {query }}}\left\langle N_{C_{\text {query }}}, C_{\text {deformed }}(f)\right\rangle \\
+\frac{1}{\lambda}\left[k_{C_{\text {query }}}-k_{C_{\text {deformed }}}(f)\right] k^{\prime} C_{\text {deformed }}(f)=0
\end{aligned}
$$

It is obvious that Equation (9) is complicated and difficult to solve. Our intention is to find a simplified equation, without losing the bending energy and smoothness requirements.

According to Yang [8].

$$
\int\left\|\frac{\partial\left(C_{\text {deformed }}\left(s^{\prime}\right)-C_{\text {query }}(s)\right)}{\partial s}\right\|^{2} d s \geq \int\left|\frac{\partial[f(s)-s]}{\partial s}\right|^{2} d s
$$

Where $f(s)-s$ is the displacement due to the deformation.

Equation (10) establishes that the newly introduced smoothness term:

$$
E_{\text {smooth }}=\int\left|\frac{\partial[f(s)-s]}{\partial s}\right|^{2} d s
$$

Writing the Euler-Lagrange equation for the variational Equation (5) using the smoothness term of Equation (11), leads to a more simple equation:

$$
\left\{\begin{array}{c}
f^{\prime \prime}+\frac{1}{\lambda}\left[k_{C_{\text {query }}}(s)-k_{C_{\text {deformed }}}(f(s))\right] k_{C_{\text {deformed }}}^{\prime}(f)=0 \\
+ \text { boundaryconditions }
\end{array}\right.
$$

\section{Similarity Measure}

After the deformed template reaches convergence over a shape image. We need to measure how much the shape in image is similar to the original template, and it is a fuzzy concept. In order to measure it, the first we need to think about is overlapping between the deformed template and the shape image. The second need to be thought about is the elastic deformation between the original template and the deformed template. An example was shown in Fig.1.

The similarity measurement in this paper is scaling, rotation, translation invariant. In Fig.2, the five-tip star template is made warp over a set of rotated star images that are also scaled up or down. From Table.1, it can be noticed that the energy of similarity measurement are fairly equal. 

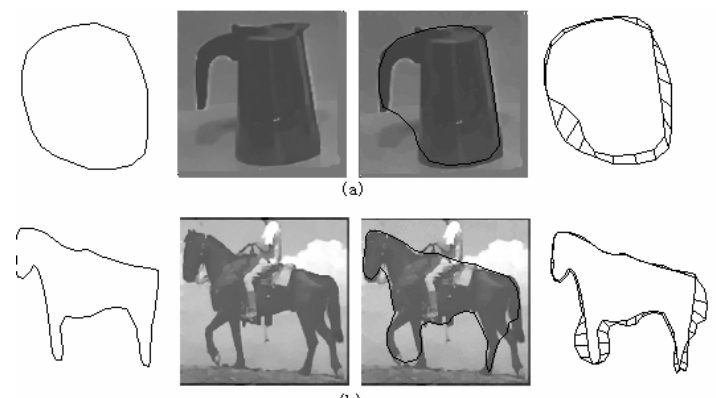

Fig. 1. Elastic matching and deformation

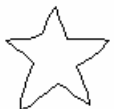

template

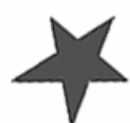

(d)

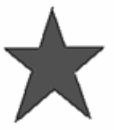

(a)



(e)

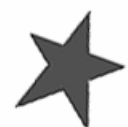

(b)

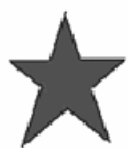

(f)

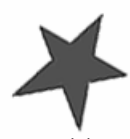

(c)

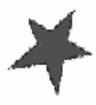

(g)

Fig. 2. Template of a five-tips star and images rotated and scaled up or down

Table 1. Energy of the star template to match the shape images in Fig.2

\begin{tabular}{cc}
\hline Star & Energy (elastic matching) \\
\hline (a) original & 0.67 \\
(b) rotated by $20^{\circ}$ & 0.62 \\
(c) rotated by $30^{\circ}$ & 0.52 \\
(d) rotated by $40^{\circ}$ & 0.63 \\
(e) scaled down & 0.61 \\
(f) scaled up & 0.68 \\
g) rotated and scaled & 0.70 \\
\hline
\end{tabular}

\section{Results}

The proposed method was implemented under the Visual C++ 6.0 system on a P41.4GHz PC. A shape database as shown in Fig.3 including nine categories with 11 shapes in each category was used to test the proposed method. In Fig.4, for each template, the 11 most similar shapes were retrieved ranked by the similarity measurement. It is clear that the approach is tolerant to occlusion although there are some missing or occluded parts in some categories such as humans, rabbits and 
hands shapes. The retrieval time was well under $3 \mathrm{~s}$ for each template to rank the 99 shapes. Fig.5 compared the \% retrieval accuracy of the proposed method with other methods such as: Curvature scale space descriptors (CSSD), Centroid-contour distance (CCD). Precision is defined as the ratio of the number of retrieved relevant shapes to the total number of retrieved shapes. Recall is defined as the ratio of the number of retrieved shapes to the total number of relevant shapes in the whole database.

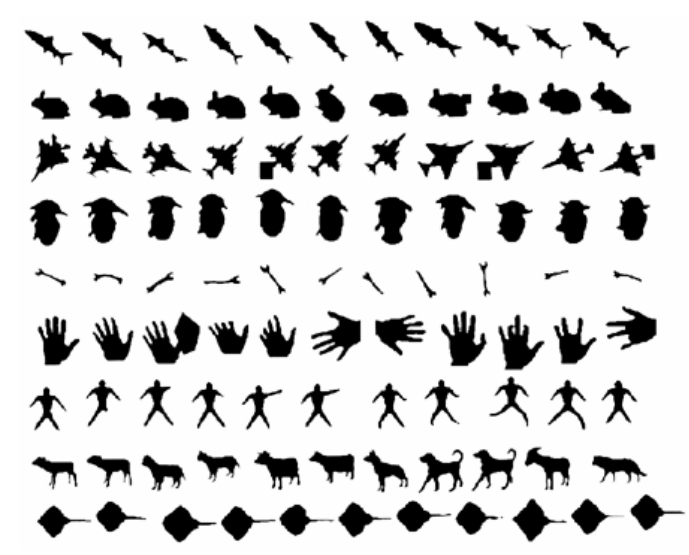

Fig. 3. Shape image database for test

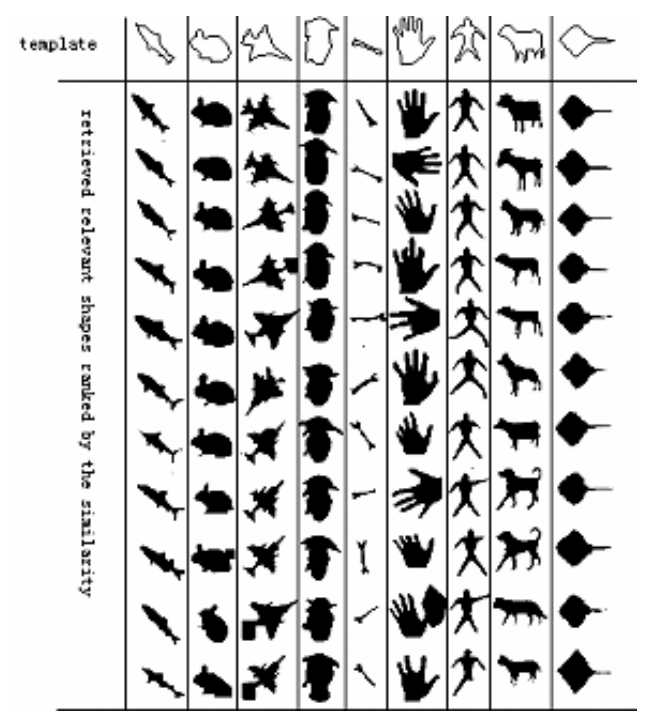

Fig. 4. Retrieval results for the shape database 


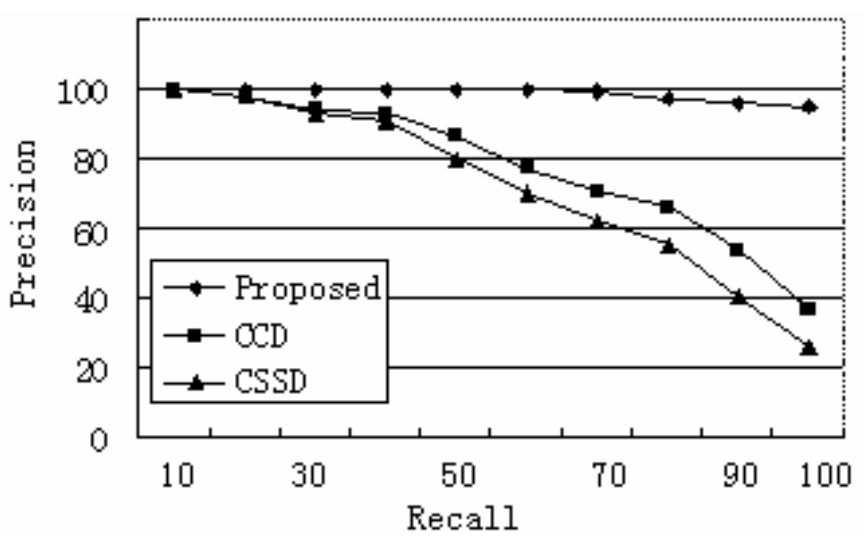

Fig. 5. Average retrieval accuracy

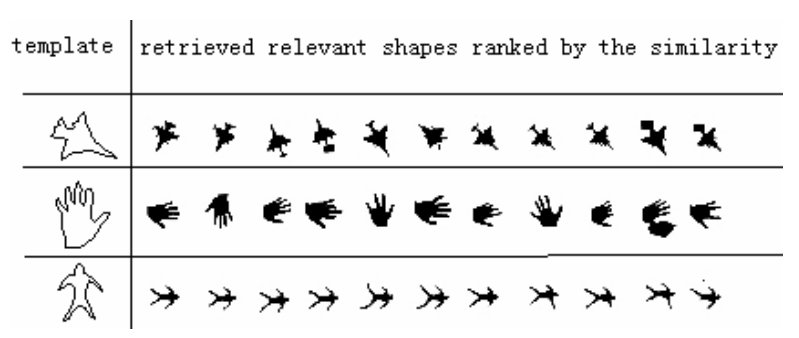

Fig. 6. Retrieval results for the shape database

More significantly, in order to demonstrate the method is scaling and rotational invariance, the experiment was repeated with the images that are scaled down to $50 \%$ and rotated by $90^{\circ}$. From Fig.6, we can see that the result is identical regardless of their scaling and rotation.

\section{Conclusions}

A new elastic matching method for shape image retrieval has been presented in this paper. Since the similarity measurement is invariant to translation, rotation and scale variations, the proposed method can handle the invariance requirement. In addition, it allows one to handle situations in which part of the shape information is missing or occluded. Finally, since no start point problem when matching shapes, the computational efficiency is not degraded. It has been proven by various experiments.

\section{Acknowledgments}

This work was partially supported by National Science Research Program of China (No. 2004BA714802) and Shanghai Science and Technology Development Foundation (034119820). 


\section{References}

1. C. Faloutsos, R. Barber,M. Flickner, J.: Efficient and effective querying by image content. J. Intell. Inform. Systems 3 (1994) 231-262

2. M. Flickner et al.: Query by image video content: The QBIC system. IEEE Comput. (1995) 28 23-32

3. S. Abbasi, F. Mokhtarian.: Enhancing CSS-based shape retrieval for objects with shallow concavities. Image Vision Comput. (2000) 18 199-211

4. Y.Gdalyahu and D.Weinshall.: Flexible syntactic matching of curves and its application to automatic hierarchical classification of silhouettes. IEEE Trans. Pattern Anal. Mach. Intell. (1999) 21 1312-1328

5. A. Del Bimbo and P. Pala.: Shape indexing by multi-scale representation. Image Vision Comput. (1999) 17 245-261

6. Kass M, Witkin A and Terzopoulos D.: Snakes: active contour models. International Journal of Computer Vision (1987) 1 321-331

7. J.S. Duncan, R. Owen, P. Anandan.: Shape-based tracking of left ventricular wall motion. Computers in Cardiology 1990, IEEE Computer Society, Chicago, Illinois, September 1990 23-26

8. Yang Xin, Bart Truyen. Hierarchical contour matching in medical images. Image and Vision Computing (1996) 14 417-433

9. Cohen I, Ayache N, Sulger P.: Tracking points on deformable curves. Proc Second Euro. Conf. Computer Vision 1992 Santa Margherita Ligure, Italy, may 1992 